PAPER

\title{
Convolutive Residual Echo Power Estimation for Acoustic Echo Reduction
}

\author{
Masahiro Fukui ${ }^{1}$, Suehiro Shimauchi ${ }^{2}$ and Akira Nakagawa ${ }^{1}$ \\ ${ }^{1}$ NTT Media Intelligence Laboratories, NTT Corporation, 3-9-11 Midori-cho, Musashino-shi, Tokyo 180-8585, Japan \\ ${ }^{2}$ Department of Electrical and Electronic Engineering, Kanazawa Institute of Technology \\ 7-1 Ohgigaoka, Nonoichi, Ishikawa 921-8501, Japan \\ E-mail: fukuimas@ieee.org
}

\begin{abstract}
This paper attempts to estimate the residual echo spectrum that results from the reverberant component beyond a length of a short-time Fourier transform block. A residual echo reduction process suppresses a residual echo signal left after an adaptive filter eliminates an acoustic echo by applying a multiplicative gain calculated from an estimated residual echo power spectrum. However, the power-spectrum estimate in a conventional method reproduces only a fraction of the echo-path impulse response, and the remaining reverberant components are only estimated roughly. Therefore, the conventional method causes large estimation errors of the reverberant components. To address this problem, this paper proposes a finite nonnegative convolution method to estimate the residual echo power spectrum. This method first estimates the power spectra of each segment of echo-impulse response. The method then calculates the residual echo power spectra corresponding to each segment of the residual echo from the power spectra of each segment of the echoimpulse response. Finally, it obtains the residual echo power spectrum that includes the late reverberant components by convoluting their estimated power spectra in a time axis direction. The proposed method estimates all the power spectra in each segment of echo-impulse response by computing cross correlation between the complex spectra of the microphone-input and received speech signals, while shifting present and past frames of the received speech signal in the time axis direction.
\end{abstract}

Keywords: hands-free teleconferencing, acoustic echo canceller, residual echo reduction, Wiener filter, late reverberation

\section{Introduction}

An acoustic echo canceller (AEC) is a digital signal processing technology used in hands-free teleconferencing. It helps to prevent howling and eliminate undesired acoustic echoes that propagate from a loudspeaker to a microphone. Most AECs use in series an adaptive filter (ADF) [1], [2] of a finite impulse response (FIR) linear filter structure and echo reduction (ER) [3]-[8] of a nonlinear post-filter. The ADF process cancels out the acoustic echo signal by adaptively modeling an unknown acoustic echo path, but some residual echo signal remains in its output (often called error signal). Thus, the ER process follows the ADF process and reduces the residual echo signal [9], [10]. This process suppresses the residual echo signal by applying a multiplicative gain, which is called an echoreduction gain, for each frequency component of the error signal. The echo-reduction gain is calculated from the estimate of the power spectrum of the echo-path impulse response (which we call the echo-path power spectrum hereafter).

Two representative methods exist to estimate the echo-path power spectrum. One is a straightforward and simple method [3], [4] that measures the echo-path power spectrum when disturbances such as a near-end speech are detected to be absent. The other is a frequency-domain cross correlation (FDCC) method [5][8] using the correlation between received speech and error signals. A major advantage of FDCC is that it can be used to estimate the echo-path power spectrum even during doubletalk when far-end and near-end speakers simultaneously talk. Therefore, the FDCC approach is gradually becoming the mainstream method. However, this approach has a problem: it reproduces only a fraction of the echo-path impulse response that mainly consists of direct sound and early reflection. Thereby, the late echo components that result from the late reverberation are not considered. This is because the time length of one block where the fast Fourier transform (FFT) is applied is usually far shorter than that of the echo-path impulse response in order to reduce the processing delay; the longer the time length of the FFT block, the more the processing delay increases.

As an ordinary method to address this problem, an exponential decay approximation model [11], [12] of the echo-path impulse response is often used in conjunction with FDCC to offset the late echo components. In this model, the late echo components are compensated for by recursively adding the estimate of the residual echo power spectrum obtained with FDCC. The added amount of the late echo components is usually adjusted in accordance with the reverberation time. An adaptive method was also proposed to estimate the reverberation 
time for the exponential decay model [11]. However, the authors found a significant problem with the exponential decay approximation model; it requires the assumption that the spectral structure of the microphone input signal in a present frame is represented by exponential decay of the spectrum in a past frame. This assumption does not necessarily hold true because a non-stationary signal such as speech is given by conversation in telecommunications. As a result, the exponential-decaymodel approach often fails to accurately estimate the residual echo power spectrum in practical reverberant environments. Moreover, this causes the sound quality to perceptually degrade after applying the ER process.

To resolve these problems, this paper proposes estimating the residual echo power spectrum not by using the exponential decay model but by assuming a finite nonnegative convolution model that convolutes each segment of echo-path impulse response with the received speech signal in the power spectral domain; it can take the non-stationarity of the signal into account. The proposed method first estimates the power spectra of each segment of echo-impulse response, second calculates the residual echo power spectra corresponding to each segment of the residual echo from the power spectra of each segment of echo-impulse response, and finally obtains the residual echo power spectrum that includes the reverberant components by convoluting their estimated power spectra in a time axis direction. With the proposed method, all the power spectra in each segment of echo-impulse response are estimated by computing the cross correlation between the complex spectra of the microphone-input and received speech signals, while shifting present and past frames of the received speech signal in a time axis direction.

The remainder of this paper is organized as follows. Section 2 presents an overview of the conventional ER method and its problems. Section 3 provides details of the proposed method. Section 4 describes the simulation and subjective experimental results, and Sec. 5 concludes this paper.

\section{Echo Reduction and Its Issues}

\subsection{Conventional echo reduction}

This section explains the ER process in a short-time spectral amplitude (STSA) domain [13] using the FDCC and exponential decay approaches. The structure of the ER process is illustrated in Fig. 1. The ADF process is omitted to simplify the explanation. Hereafter, the residual echo signal is treated as the microphone input signal. The microphone input signal $y(k)$ is expressed as

$$
y(k)=d(k)+s(k)
$$

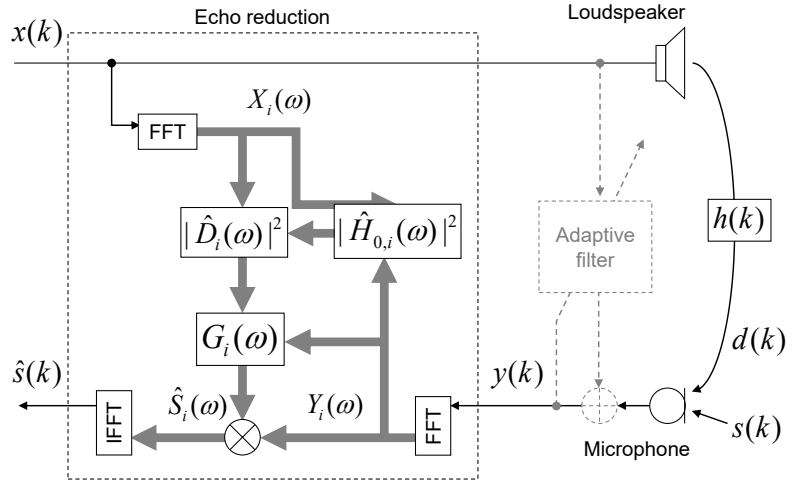

Fig. 1 Structure of echo reduction process

where $k$ is a sample number of discrete time; $d(k)$ and $s(k)$ are acoustic echo and near-end speech signals, respectively. The acoustic echo signal $d(k)$ is represented by a convolution of an echo-path impulse response $h(k)$ and a received speech signal $x(k)$, i.e.,

$$
d(k)=h(k) * x(k)
$$

where * denotes the convolution. The short-time spectrum of $y(k)$ is represented as

$$
Y_{i}(\omega)=D_{i}(\omega)+S_{i}(\omega)
$$

where $\omega$ is a discrete frequency index, $i$ is a discrete time-frame index, and $S_{i}(\omega)$ is the short-time spectrum of $s(k)$, respectively. The echo spectrum, $D_{i}(\omega)$, which is the short-time spectrum of the echo signal $d(k)$, is expressed by the convolution of the echo path $h(k)$ and the received speech signal $x(k)$ in the frequency domain [14] as follows:

$$
D_{i}(\omega)=\sum_{m=0}^{\infty} H_{m, i}(\omega) X_{i-m}(\omega)
$$

where $m$ denotes the segment index. $H_{m, i}(\omega)$ is the short-time spectrum obtained from a fraction of the length of $h(k) ; H_{0, i}(\omega)$ is the spectrum of the early part of $h(k)$ that includes the direct sound and early reflections (commonly called early response). The 
remaining portions $\left(H_{m, i}(\omega) ; m>0\right)$ are the spectra of the late reverberation. The relationship between $h(k)$ and $H_{m, i}(\omega)$ is illustrated in Fig. 2.

The echo reduction can be expressed using an echoreduction gain $G_{i}(\omega)$ in a general form as follows:

$$
\hat{S}_{i}(\omega)=G_{i}(\omega) Y_{i}(\omega)
$$

where $\hat{S}_{i}(\omega)$ denotes the estimate of $S_{i}(\omega)$. $G_{i}(\omega)$ is for example calculated by a Wiener filtering method [15], [16] obtained by the following equation:

$$
G_{i}(\omega)=\frac{\left|Y_{i}(\omega)\right|^{2}-\left|\hat{D}_{i}(\omega)\right|^{2}}{\left|Y_{i}(\omega)\right|^{2}}
$$

where $\left|\hat{D}_{i}(\omega)\right|^{2}$ is the estimate of echo power spectrum $\left|D_{i}(\omega)\right|^{2}$. The obtained estimate $\hat{S}_{i}(\omega)$ is transformed into the time domain signal $\hat{s}(k)$, which is the send signal, by an inverse FFT (IFFT).

The echo power spectrum is estimated using the exponential decay model as

$$
\begin{aligned}
\left|\hat{D}_{i}(\omega)\right|^{2} & =\left|\hat{H}_{0, i}(\omega)\right|^{2}\left|X_{i}(\omega)\right|^{2}+\alpha\left|\hat{D}_{i-1}(\omega)\right|^{2} \\
& =\left|\hat{D}_{0, i}(\omega)\right|^{2}+\alpha\left|\hat{D}_{i-1}(\omega)\right|^{2}
\end{aligned}
$$

where $\alpha$ is a design parameter to control the reverberation time; it determines the amount of added late echo components. $0 \leq \alpha \leq 1$ is used in accordance with the reverberation time. For instance, $\alpha$ is set at 0.72 when the frame shift size is $8 \mathrm{~ms}$ and the reverberation time with an RT60 [17] is $160 \mathrm{~ms}$. A variable $\left|\hat{D}_{0, i}(\omega)\right|^{2}$ is the estimate of the echo power spectrum corresponding to the early response; $\left|\hat{H}_{0, i}(\omega)\right|^{2}$ denotes the power-spectrum estimate of $H_{0, i}(\omega)$; it is given by

$$
\left|\hat{H}_{0, i}(\omega)\right|^{2}=\left|\frac{\left\langle\mathbf{X}_{i}(\omega), \mathbf{Y}_{i}(\omega)\right\rangle}{\left\|\mathbf{X}_{i}(\omega)\right\|^{2}}\right|^{2}
$$

where $\langle$,$\rangle and \|\cdot\|$ are an inner product and a norm,

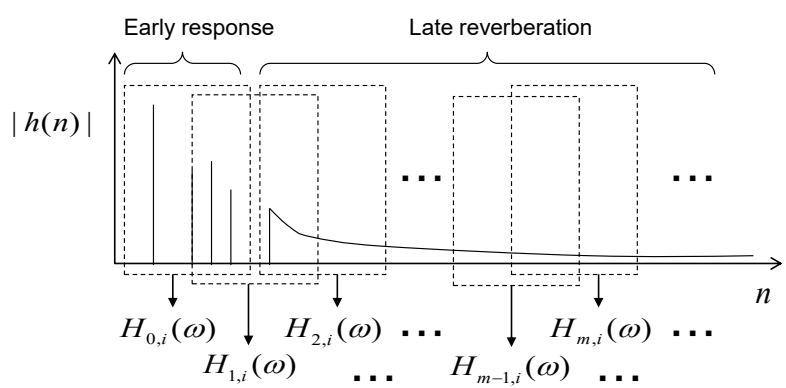

Fig. 2 Relationship between echo-path impulse response and its short-time spectra in overlap-add FFT processing

respectively. Boldface denotes a time-sequence vector of a short-time spectrum; it is defined as: $\mathbf{P}_{i}(\omega)=\left[P_{i}(\omega), \cdots, P_{i-L+1}(\omega)\right]^{T} . L$ is the number of frames, meaning the observation time parameter, and $T$ is a transposition. Given the time-sequence vector of the microphone input spectrum as a sum of the statistically independent echo and near-end speech spectra $\mathbf{Y}_{i}(\omega)=\mathbf{D}_{i}(\omega)+\mathbf{S}_{i}(\omega)$, then the estimate of the echo-path power spectrum in Eq. (8) is finally obtained from the following relationship:

$$
\begin{aligned}
\left|\hat{H}_{0, i}(\omega)\right| & =\left|\frac{\left\langle\mathbf{X}_{i}(\omega), \mathbf{Y}_{i}(\omega)\right\rangle}{\left\|\mathbf{X}_{i}(\omega)\right\|^{2}}\right|^{2} \\
& =\left|\frac{\left\langle\mathbf{X}_{i}(\omega), \mathbf{D}_{i}(\omega)+\mathbf{S}_{i}(\omega)\right\rangle}{\left\|\mathbf{X}_{i}(\omega)\right\|^{2}}\right|^{2} \\
& =\left|\frac{\left\langle\mathbf{X}_{i}(\omega), \mathbf{D}_{i}(\omega)\right\rangle+\left\langle\mathbf{X}_{i}(\omega), \mathbf{S}_{i}(\omega)\right\rangle}{\left\|\mathbf{X}_{i}(\omega)\right\|^{2}}\right|^{2} \\
& \approx\left|\frac{\left\langle\mathbf{X}_{i}(\omega), \mathbf{D}_{i}(\omega)\right\rangle}{\left\|\mathbf{X}_{i}(\omega)\right\|^{2}}\right|^{2} \\
& \approx\left|\frac{H_{i}(\omega)\left\langle\mathbf{X}_{i}(\omega), \mathbf{X}_{i}(\omega)\right\rangle}{\left\|\mathbf{X}_{i}(\omega)\right\|^{2}}\right|^{2} \\
& =\left|H_{i}(\omega)\right|^{2}
\end{aligned}
$$

\subsection{Problem with exponential decay model}

The exponential decay approximation model simulates the echo power spectrum by the following 
approximate expansions:

$$
\begin{aligned}
\left|\hat{D}_{i}(\omega)\right|^{2} & =\left|\sum_{m=0}^{\infty} \hat{H}_{m, i}(\omega) X_{i-m}(\omega)\right|^{2} \\
& \approx \sum_{m=0}^{\infty}\left|\hat{H}_{m, i}(\omega)\right|^{2}\left|X_{i-m}(\omega)\right|^{2} \\
& \approx \sum_{m=0}^{\infty} \alpha^{m}\left|\hat{H}_{0, i}(\omega)\right|^{2}\left|X_{i-m}(\omega)\right|^{2} \\
& =\left|\hat{D}_{0, i}(\omega)\right|^{2}+\sum_{m=1}^{\infty} \alpha^{m}\left|\hat{H}_{0, i}(\omega)\right|^{2}\left|X_{i-m}(\omega)\right|^{2} \\
& =\left|\hat{D}_{0, i}(\omega)\right|^{2}+\alpha \sum_{m=0}^{\infty} \alpha^{m}\left|\hat{H}_{0, i}(\omega)\right|^{2}\left|X_{i-m-1}(\omega)\right|^{2} \\
& =\left|\hat{D}_{0, i}(\omega)\right|^{2}+\alpha\left|\hat{D}_{i-1}(\omega)\right|^{2}
\end{aligned}
$$

The late echo components are modelled as $\alpha\left|\hat{D}_{i-1}(\omega)\right|^{2}$ by the above approximate expansion: $\left|\hat{H}_{m, i}(\omega)\right|^{2} \approx \alpha^{m}\left|\hat{H}_{0, i}(\omega)\right|^{2}$. However, if this assumption holds true, the following relationships between $\left|H_{m, i}(\omega)\right|^{2}$ and $\left|H_{0, i}(\omega)\right|^{2}$ must hold:

$$
\begin{aligned}
\left|\hat{H}_{m, i}(\omega)\right|^{2} & \approx \alpha^{m}\left|\hat{H}_{0, i}(\omega)\right|^{2} \\
& =\alpha^{m}\left|\frac{\left\langle\mathbf{X}_{i}(\omega), \mathbf{Y}_{i}(\omega)\right\rangle}{\left\|\mathbf{X}_{i}(\omega)\right\|^{2}}\right|^{2} \\
& =\alpha^{m}\left|\frac{\left\langle\mathbf{X}_{i-m}(\omega), \mathbf{Y}_{i-m}(\omega)\right\rangle}{\|\left.\mathbf{X}_{i-m}(\omega)\right|^{2}}\right|^{2} \\
& =\left|\frac{\left\langle\mathbf{X}_{i-m}(\omega), \alpha^{\frac{m}{2}} \mathbf{Y}_{i-m}(\omega)\right\rangle}{\|\left.\mathbf{X}_{i-m}(\omega)\right|^{2}}\right|^{2} \\
& \approx\left|\frac{\left\langle\mathbf{X}_{i-m}(\omega), \mathbf{Y}_{i}(\omega)\right\rangle}{\left\|\mathbf{X}_{i-m}(\omega)\right\|^{2}}\right|^{2} \\
& =\left|\hat{H}_{m, i}(\omega)\right|^{2}
\end{aligned}
$$

where

$$
\begin{aligned}
\left|\hat{H}_{0, i}(\omega)\right|^{2} & =\left|\hat{H}_{0, i-m}(\omega)\right|^{2} \\
& =\left|\frac{\left\langle\mathbf{X}_{i-m}(\omega), \mathbf{Y}_{i-m}(\omega)\right\rangle}{\left\|\mathbf{X}_{i-m}(\omega)\right\|^{2}}\right|^{2}
\end{aligned}
$$

if the echo-path impulse response remains unchanged.

The approximate expansions of Eq. (11) are based on the premise that the spectral structure of the microphone input signal in the present frame is represented by exponential decay of the spectrum in the past frame: $\mathbf{Y}_{i}(\omega)=\alpha^{\frac{m}{2}} \mathbf{Y}_{i-m}(\omega)$. However, an exponentially decaying microphone input is unrealistic; because in most cases, the microphone picks up the non-stationary signal such as speech not held true to this assumption. Consequently, the conventional approach suffers from the speech distortions of the send signal after processing due to the echo power spectrum being inaccurately estimated.

\section{Proposed Echo Power Estimation}

This section presents a method to improve the accuracy of the conventional echo power spectral estimation. First, the proposed method estimates the power spectra of each segment of the echo-impulse response. Second, this method calculates the estimates of the echo power spectra corresponding to each segment of the acoustic echo signal using the estimated power spectra of each segment of the echo-impulse response; and the method computes the estimate of the echo power spectrum including all the reverberant components of the echo-impulse response.

The power spectra in each segment of the echoimpulse response, $\left|H_{0, i}(\omega)\right|^{2}, \cdots,\left|H_{M-1, i}(\omega)\right|^{2}$, are estimated by computing cross correlation between the complex spectra of the microphone-input and received speech signals, respectively, while shifting the present and past frames of the received speech signal in a time axis direction. Here $M$ is the number of segments equivalent to the reverberation time. Specifically, the estimate of the power spectra in segment $m$ of echoimpulse response, $\left|\hat{H}_{m, i}(\omega)\right|^{2}$, is calculated from

$$
\left|\hat{H}_{m, i}(\omega)\right|^{2}=\left|\frac{\left\langle\mathbf{X}_{i-m}(\omega), \mathbf{Y}_{i}(\omega)\right\rangle}{\left\|\mathbf{X}_{i-m}(\omega)\right\|^{2}}\right|^{2}
$$

By using the obtained $\left|\hat{H}_{0, i}(\omega)\right|^{2}, \cdots,\left|\hat{H}_{M-1, i}(\omega)\right|^{2}$ in Eq. (13) and the received speech power spectrum 
$\left|X_{i}(\omega)\right|^{2}$, the estimate of the echo spectrum is derived from a finite nonnegative convolution equation in the power spectral domain; it is given as

$$
\begin{aligned}
\left|\hat{D}_{i}(\omega)\right|^{2} & =\sum_{m=0}^{M-1}\left|\hat{H}_{m, i}(\omega)\right|^{2}\left|X_{i-m}(\omega)\right|^{2} \\
& =\sum_{m=0}^{M-1}\left|\hat{D}_{m, i}(\omega)\right|^{2}
\end{aligned}
$$

In the proposed method, the estimate of the echo power spectrum of the segment $m$ are represented with $\left|\hat{D}_{m, i}(\omega)\right|^{2}=\left|\hat{H}_{m, i}(\omega)\right|^{2}\left|X_{i-m}(\omega)\right|^{2}$. The necessary number of segment $M$ is determined in accordance with the reverberation time. For example, it is set at 20 when the frame shift size is $8 \mathrm{~ms}$ and the reverberation time is $160 \mathrm{~ms}$.

As can be seen from Eq. (14), the estimated echo power spectrum is composed of the estimate of the echo component corresponding to the early response $\left|\hat{D}_{0, i}(\omega)\right|^{2}$ and segments of the estimates of the late echo components $\left|\hat{D}_{1, i}(\omega)\right|^{2}, \cdots,\left|\hat{D}_{M-1, i}(\omega)\right|^{2}$. The merit of this method is it can deal with the nonstationarity of the microphone input signal unlike the conventional exponential decay approximation model that simply approximates the late echo components through the weighted additive type; because the late echo components can be derived from Eq. (13) as with the echo power spectrum corresponding to the early response. Although Eq. (14) with the convolutive type has a demerit that it is more complicated to calculate than Eq. (7) of the conventional exponential decay model, in the proposed method, a significant improvement in the estimation accuracy of the late echo components can be expected against the non-stationary microphone input signal for the above-described reasons.

Also, the ER process often reconstructs the send signal by overlapping and adding the windowed output frames. To reduce the computational complexity of the proposed method, if the FFT window overlap of $50 \%$ is adopted in the ER process, the method enables us to omit some calculations and estimate the echo power spectrum as follows:

$$
\left|\hat{D}_{i}(\omega)\right|^{2}=\sum_{m=0}^{M / 2-1}\left|\hat{H}_{2 m, i}(\omega)\right|^{2}\left|X_{i-2 m}(\omega)\right|^{2}
$$

\section{Experiments}

The performance of the proposed method was evaluated using both simulation and subjective listening tests. The conventional and proposed methods were applied to calculate the echo power spectrum using Eqs. (7) and (15), respectively. Table 1 lists the experimental conditions. In these experiments, the ADF process was omitted.

\subsection{Comparison of echo-power estimation accuracy}

The echo-power estimation errors of the conventional and proposed methods are shown in Fig. 3. The estimation error is represented using a signal-todistortion ratio (SDR) [18] as the difference between the target and estimated echo-power spectra in a received single-talk period that only the far-end speaker talks; the SDR is defined as

$$
\mathrm{SDR}=10 \log _{10} \frac{\sum_{\omega}\left|D_{i}(\omega)\right|^{2}}{\sum_{\omega} \max \left\{\left|D_{i}(\omega)\right|^{2}-\left|\hat{D}_{i}(\omega)\right|^{2}, 0\right\}}
$$

These sound qualities after processing were compared under reverberation times of 50, 100, 150, 250, 350, 500, and $750 \mathrm{~ms}$. The female talker signal digitized at a sampling rate of $16 \mathrm{kHz}$ was used as the sound source of the received speech signal. The language is English. The design parameter $\alpha$ was set at $0.3,0.55,0.7,0.8,0.85$, 0.9 , and 0.93 , respectively, in accordance with the reverberation time. The number of segments $M$ was set at 20 for all the conditions of the reverberation-times. As Fig. 3 indicates, the proposed echo-power estimation method improved the estimation accuracy under all these reverberation time conditions.

\subsection{Comparison of echo reduction performance}

This subsection evaluates the performance of the proposed echo-power estimation method using simulation tests to compare it with the conventional method (exponential decay model). The reverberation time is $160 \mathrm{~ms}$ in this simulation. The design parameter $\alpha$ was set to 0.72 ; and the number of segments $M$ was set at 20 .

The received and near-end speech signals are shown in Figs. 4 and 5, respectively. Periods A and B are the received and send single-talk periods, respectively. The send single-talk period means the period that only the near-end speaker talks. A double-talk period occurs during a period $\mathrm{C}$. The microphone input signal $y(k)$ is shown in Fig. 6. Figures 7 and 8 plot the send signals $\hat{s}(k)$ after the conventional and proposed methods were respectively applied. As seen in Figs. 6, 7, and 8, the conventional and proposed methods sufficiently 
Table 1 Experimental conditions

\begin{tabular}{l|l}
\hline Category & Value \\
\hline Sampling rate & $16 \mathrm{kHz}$ \\
Frame length & 256 samples \\
Frame shift & 128 samples \\
FFT points & 256 samples \\
Signal length & $12 \mathrm{~s}$ \\
\hline
\end{tabular}

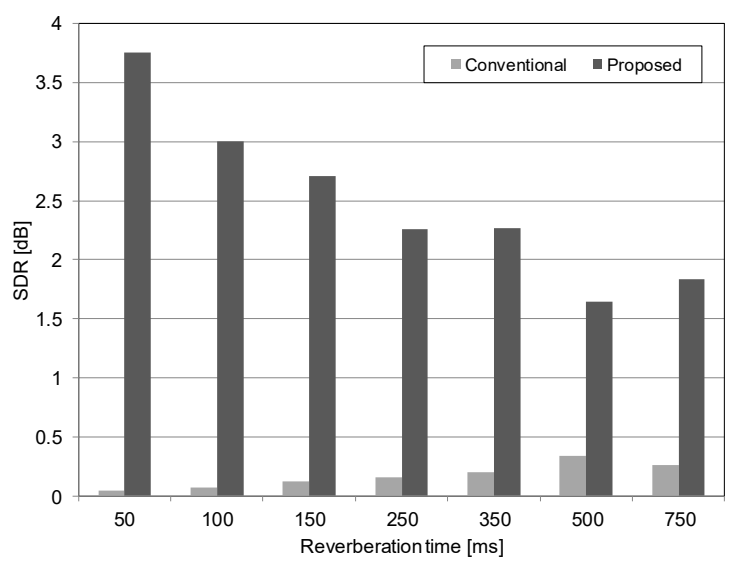

Fig. 3 Comparison of SDR during received single-talk period
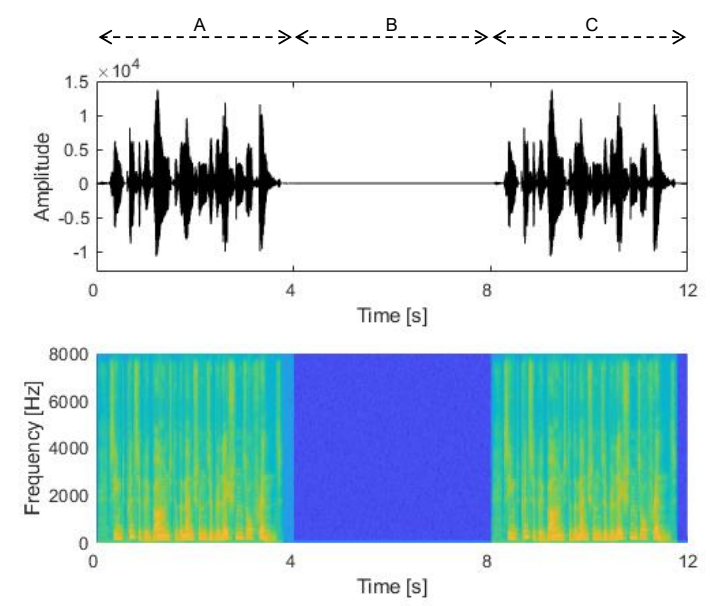

Fig. 4 Received speech signal (female speech)

suppressed the echo signals in the received single-talk period A. An echo return loss enhancement (ERLE) [19] was used to measure the echo-suppression level; the ERLE is defined as

$$
\mathrm{ERLE}=10 \log _{10} \frac{p_{\text {in }}}{p_{\text {out }}}
$$
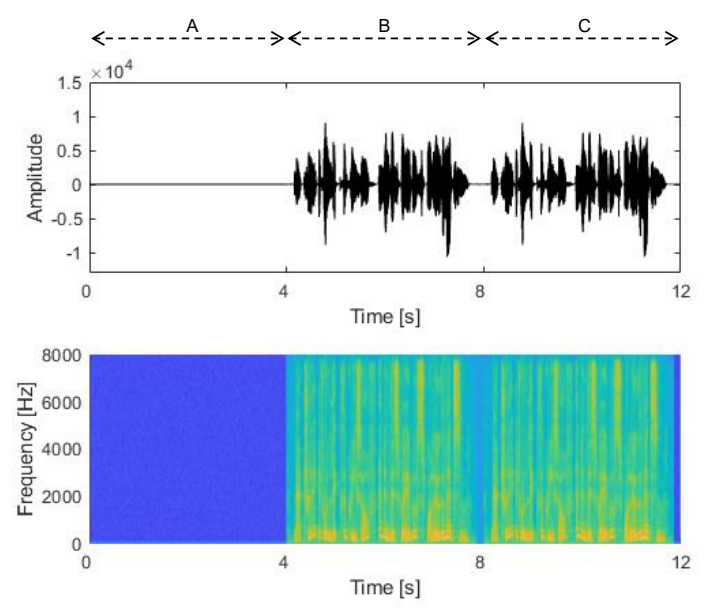

Fig. 5 Near-end speech signal (male speech)
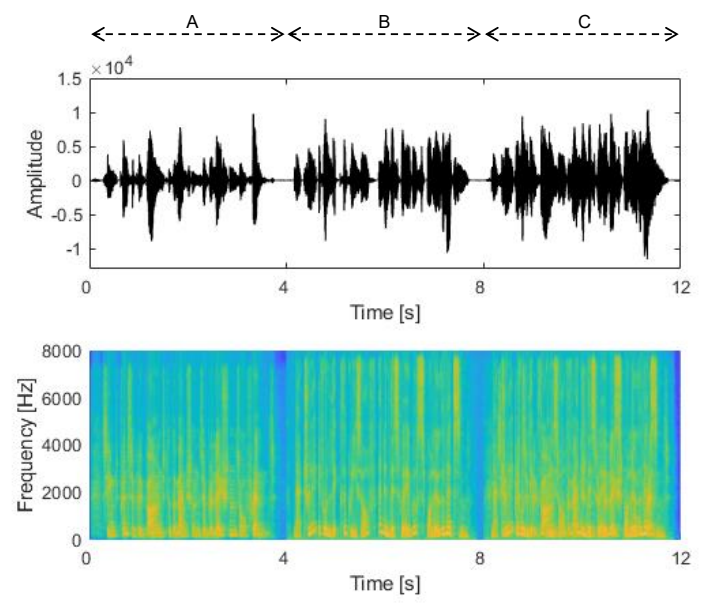

Fig. 6 Microphone signal

where $p_{\text {in }}$ and $p_{\text {out }}$ denote mean squares of the microphone input and send signals during the received single-talk periods A and B. The ELREs of 22.98 and $28.19 \mathrm{~dB}$ were achieved as the echo-suppression levels using the conventional and proposed methods, respectively. However, these results show that speech distortions seemed to occur during the double-talk period $\mathrm{C}$ especially when the conventional method was applied.

The amount of speech distortion during the doubletalk period $\mathrm{C}$ was evaluated using a linear predictive coding (LPC) cepstral distance [20], which is computed as follows:

$$
\mathrm{CD}(k)=\frac{10}{\log 10} \sqrt{2 \sum_{m=1}^{16}[c(m, k)-\hat{c}(m, k)]^{2}}
$$



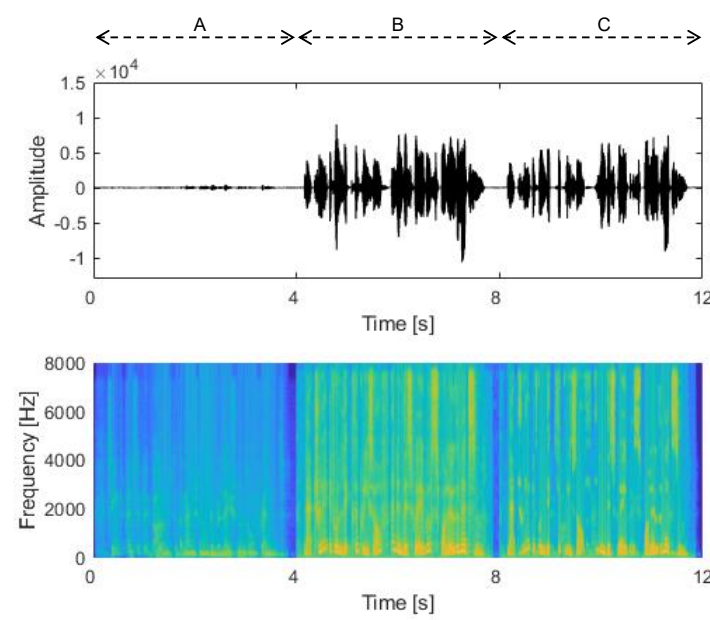

Fig. 7 Send signal with conventional method
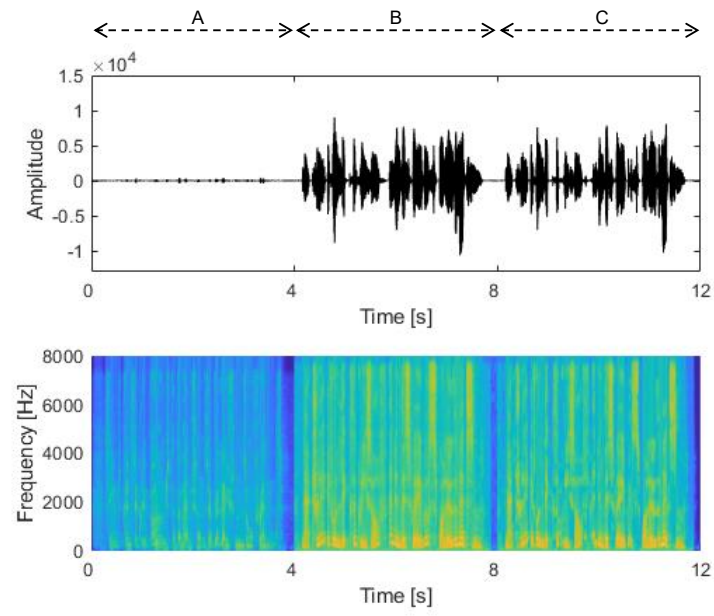

Fig. 8 Send signal with proposed method

where $c(m, k)$ and $\hat{c}(m, k)$ are the $k$-th cepstral coefficients of near-end speech and send signals, respectively, and $\mathrm{CD}(k)$ is the LPC cepstral distance.

The results of comparing the conventional and proposed methods are shown in Fig. 9. The average cepstral distance scores of the conventional and proposed methods were 4.88 and 1.90 points, respectively. In particular, the proposed method improved sound quality by a maximum of 6.83 points in a period of about $9.2 \mathrm{~s}$ over the conventional method. Better scores were observed over almost the entire period, and significant improvement was confirmed.

\subsection{Subjective experiments}

A multi-stimulus test with hidden reference and

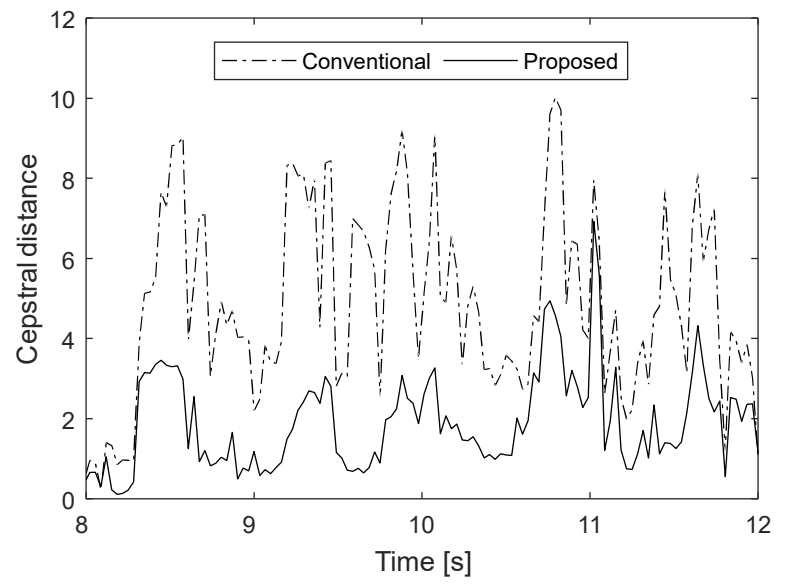

Fig. 9 Comparison of LPC cepstrum distances during double-talk situation in period $\mathrm{C}$

anchor (MUSHRA) using a 100-point scale, compliant with ITU-R BS.1534-1 [21], was used to test the quality of speech. All reference and evaluation signals were played to both ears through headphones (Sennheiser HD 280 Pro). Eight experienced listeners evaluated speech under six conditions: the near-end speech signal (c00: hidden reference), the near-end speech signal filtered by a $3.5-\mathrm{kHz}$ low-pass filter (c01: anchor $\mathrm{A}$ ), the microphone input signal (c02: anchor $\mathrm{B})$, the target signal of ER (c03: anchor C), and the send signals of conventional and proposed methods (c04: conventional method and c05: proposed method). The target signal $\hat{\mathrm{s}}_{\mathrm{T}}(k)$ denotes the limiting value of the ER process, which is simulated using the following equation:

$$
\hat{\mathbf{s}}_{T}(k)=\operatorname{IFFT}\left[\left|\mathrm{S}_{\mathrm{i}}(\omega)\right| e^{j \theta_{Y}}\right]
$$

where IFFT[.] and $\theta_{Y}$ denote the IFFT operation and the phase component of $Y_{i}(\omega)$, respectively.

The MUSHRA test results comparing the conventional and proposed methods during the doubletalk situation of the period $\mathrm{C}$ are shown in Fig. 10. The vertical lines denote a $95 \%$ confidence interval. For the double-talk period $\mathrm{C}$, mean scores were awarded for six sound signals by eight listeners. As these results indicate, a better score was observed in the double-talk period by using the proposed method that calculates the echo power spectrum by assuming a finite nonnegative convolution model. The proposed method improved the sound quality by about 6 points on a 100-point scale compared with the conventional method, and a significant improvement was confirmed. 


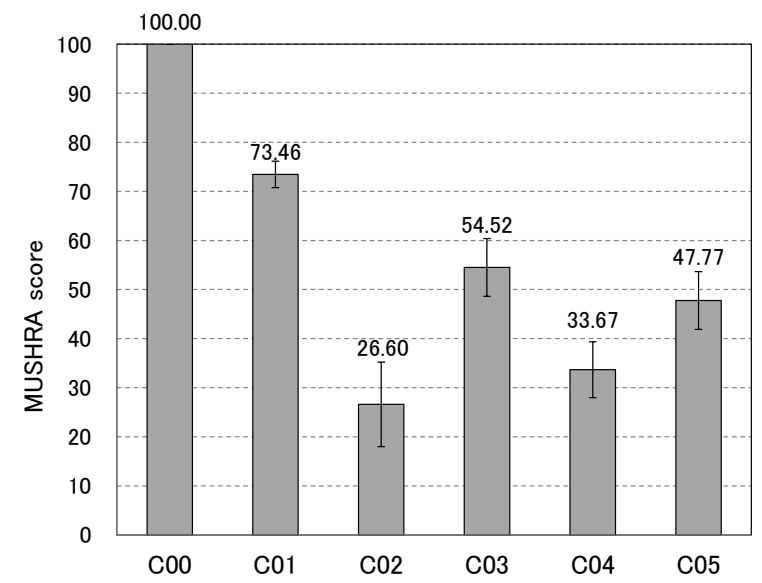

Fig. 10 Double-talk quality assessments for period C

\section{Conclusions}

A method was proposed to estimate the residual echo power spectrum for the echo reduction. The residual echo power spectrum was calculated using the finite nonnegative convolution model. It convolutes each segment of echo-path impulse response with received speech signal in the power spectral domain. The proposed method properly suppressed residual echo and distorted marginal speech in the send signal during the double-talk period. The experimental results revealed that the proposed method outperformed the conventional exponential decay model approach and improved the speech quality.

\section{References}

[1] C. Breining, P. Dreiscitel, E. Hansler, A. Mader, B. Nitsch, H. Puder, T. Schertler, G. Schmidt and J. Tilp: Acoustic echo control: An application of veryhigh-order adaptive filters, IEEE Signal Processing Magazine, Vol.16, No.4, pp.42-69, 1999.

[2] S. Shimauchi, Y. Haneda and A. Kataoka: A robust NLMS algorithm for acoustic echo cancellation, IEICE Trans. Fundamentals, Vol.J89-A, No.8, pp. 926-934, 2005.

[3] E. Hansler and G. U. Schmidt: Hands-free telephones - Joint control of echo cancellation and postfiltering, Signal Processing, Vol.80, pp.11, pp. 2295-2305, 2000.

[4] C. Avendano: Acoustic echo suppression in the STFT domain, Proc. IEEE Workshop on Applications of Signal Processing to Audio and Acoustics, Vol.21, No.24, pp.175-178, 2001.

[5] C. Faller and C. Tournery: Robust acoustic echo control using a simple echo path model, Proc. IEEE International Conference on Acoustics, Speech, and
Signal Processing, Vol.5, pp.281-284, 2006.

[6] Y. S. Park and J. H. Chang: Frequency domain acoustic echo suppression based on soft decision, IEEE Signal Processing Letters, Vol.16, No.1, pp. 53-56, 2009.

[7] M. Fukui, S. Shimauchi, Y. Hioka, A. Nakagawa and Y. Haneda: Double-talk robust acoustic echo cancellation for CD-quality hands-free videoconferencing system, IEEE Trans. Consumer Electron., Vol.60, No. 3, pp.468-475, 2014

[8] Y. Tong and Y. Gu: A modified a priori SER for acoustic echo suppression using Wiener filter, Proc. IEEE International Workshop on Acoustic Signal Enhancement, pp.1-4, 2016.

[9] C. Beaugeant, V. Turbin, P. Scalart and A. Gilloire: New optimal filtering approaches for hands-free telecommunication terminals, Signal Processing, Vol.64, No.1, pp.33-47,1998.

[10] S. Sakauchi, A. Nakagawa, Y. Haneda and A. Kataoka: Implementing and evaluating of an audio teleconferencing terminal with noise and echo reduction: Proc. IEEE International Workshop on Acoustic Echo and Noise Control, pp.191-194, 2003.

[11]A. Favrot, C. Faller and F. Kuech: Modeling late reverberation in acoustic echo suppression, Proc. IEEE International Workshop on Acoustic Signal Enhancement, pp.1-4, 2012.

[12] S. Leglaive, R. Badeau and G. Richard: Autoregressive moving average modeling of late reverberation in the frequency domain, Proc. European Signal Processing Conference, pp.14781482, 2016.

[13] S. F. Boll: Suppression of acoustic noise in speech using spectral subtraction, IEEE Trans. Acoust. Speech Signal, Processing, Vol.ASSP-27, No.2, pp. 113-120, 1979.

[14]H. Kanai, T. Hori, N. Chubachi and T. Ono: Delayed block transfer function in the frequency domain, IEEE Trans. Signal Processing, Vol.42, No.7, pp. 1669-1684, 1994.

[15] J. S. Lim and A. V. Oppenheim: Enhancement and bandwidth compression of noisy speech, Proc. IEEE, Vol.67, No.12, pp.1586-1604, 1979.

[16]R. L. B. Jeannes, P. Scalart, G. Fauco'n and C. Beaugeant: Combined noise and echo reduction in handsfree systems: A survey, IEEE Trans. Speech Audio Processing, Vol.9, No.8, pp.808-820, 2001.

[17]ISO 3382-2: Acoustics — Measurement of room acoustic parameters - Part 2: Reverberation time in ordinary rooms, International Organization for Standardization, Geneva, Switzerland, 2008.

[18] E. Vincent, H. Sawada, P. Bofill, S. Makino and J. P. Rosca: First stereo audio source separation evaluation campaign: Data, algorithms and results, Proc. International Congress on Acoustics, pp.552-559, 2007. 
[19] S. Sakauchi, Y. Haneda and A. Kataoka: Gain emphasis method for echo reduction based on a short-time spectral amplitude estimation, IEICE Trans. Fundamentals, Vol.J88-A, No.6, pp.695-703, 2005. (in Japanese)

[20] A. H. Gray Jr. and J. D. Markel: Distance measures for speech processing, IEEE Trans. Acoustics Speech Signal Processing, Vol.24, No.5, pp.380-391, 1976.

[21]ITU-R Recommendation BS.1534-1: Method for the subjective assessment of intermediate quality level of coding systems, International Telecommunications Union, Geneva, Switzerland, 2003.

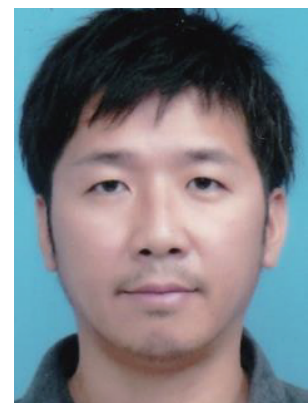

Masahiro Fukui received his B.E., M.E., and Ph.D. degrees in information science from Ritsumeikan University, Shiga, Japan, in 2002, Nara Institute of Science and Technology, Nara, Japan, in 2004, and Ritsumeikan University in 2018. Since joining NTT in 2004, he has been engaged in research on acoustic echo cancellers and speech coding. He is now a senior research engineer at NTT Media Intel-ligence Laboratories. He received the best paper award of ICCE conference and the technical development award from the Acoustic Society of Japan (ASJ) in 2014. He is a member of the Institute of Electrical and Electronics Engineers (IEEE) and ASJ.

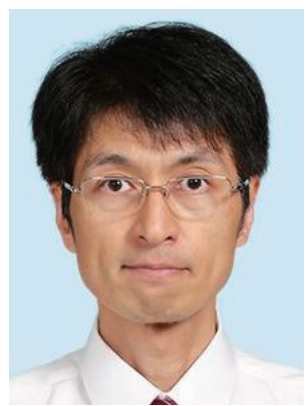

Suehiro Shimauchi received B.E., M.E., and $\mathrm{Ph} . \mathrm{D}$. degrees from Tokyo Institute of Technology in 1991, 1993, and 2007. From 1993 to 2018, he was with NTT, Tokyo, Japan. In 2018, he joined the Kanazawa Institute of Technology, Ishikawa, Japan, where he is currently a Professor in the Department of Electrical and Electronic Engineering. He has been engaged in research on acoustic signal processing and biomedical signal processing. He is a senior member of IEEE, and a member of the Institute of Electronics, Information, and Communication Engineers (IEICE) and the ASJ.

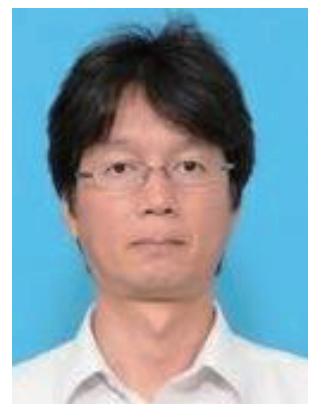

Akira Nakagawa received his B.E. and M.E. degrees from Kyushu Institute of Technology in 1992 and 1994, respectively. Since joining NTT in 1994, he has been investigating acoustic signal processing and acoustic echo cancellers. He is now a senior research engineer, supervisor at NTT Media Intelligence Laboratories. He received a paper award from the ASJ in 2001. He is a member of ASJ.

(Received November 26, 2019; revised April 20, 2020) 